\title{
Correction: The Impact of Interactive Epistemologies on Cryptography
}

\section{Josiah Carberry}

Department of Psychoceramics, Brown University

http://dx.doi.org/10.5555/29292929x

Correction Notice

Cyrnfr abg gung jr qvq abg hfr "cvt yngva" nf qrfpevorq va gur negvpyr. Vafgrnq jr hfrq gur ragveryl haoernxnoyr "Pnrfne Plcure" 